\title{
Preoperative cognitive performance, age and the choice of the main anaesthetics influence the Area Under the Curve (AUC) of intra-operative cerebral burst suprression (BS) as measured by the depth-of-anaesthesia monitor NeuroSENSE $\circledR$
}

\section{Background and goal of the study}

Studies suggest that intra-operative cerebral BS $^{1}$, preoperative cognitive impairment and advanced age are risk factors for postoperative delirium and cognitive decline ${ }^{2}$. We Hypothesized that preoperative cognitive impairment and advanced age increase brain vulnerability to the anaesthetics resulting in an increased BS despite a constant level of anaesthesia. We further analysed whether the choice of main anaesthetics could influence this vulnerability.

\begin{abstract}
Material and methods
This is a subanalysis of a prospective observational trial (NCT02006212). Only subjects undergoing normothermic cardiac surgery were analysed. Depth of anaesthesia (DOA) was maintained between $40-60$ based on NeuroSENSE $®$. BS is presented as total AUC of EEG suppression ratio > 0 seconds $\left(A \cup C_{>0 s}\right)$ and expressed in minutes\%. $A \cup C_{>0 s}$ of $>10$ minutes\% (dichotomous variable) is defined as abnormal to exclude eventual immediate postinduction BS. Binary regression analysis was performed to predict $\mathrm{AUC}_{>0 \mathrm{~s}}$ of $>10$ minutes\%. Age, baseline Mini Mental State Examination (MMSE) and the choice of anaesthetic (Sevoflurane vs Propofol) were used as independent variables. Data are expressed as median (P25-P75) or numbers and percentages. A Mann-Whitney test was used to compare continuous variables.
\end{abstract}

\section{Results and discussion}

In total 925 Patients were analysed. Patients with AUC $>0$ s of 10 minutes $\%$ had significantly lower baseline MMSE $(P=0,000)$.

Table 1: Data of the entire group

\begin{tabular}{c} 
Propofol \\
Age (years) \\
Preop MMSE \\
EuroSCORE II \\
Gender Male \\
Carotid stenosis, R \\
Carotid stenosis, L \\
\hline AUC of BS > 10 min\%, R \\
AUC of BS > 10 min\%, \\
Total AUC $>$ os (min\%), R \\
Min \\
Max \\
Total AUC $>$ os (min\%), L \\
Min \\
Max
\end{tabular}

CPB time, $\min$

Aortic cross-clamp time, min Intrahospital mortality

\section{$N=925$}

$255(27,6 \%)$

$68(58-77)$

$28(26-29)$

$1,92(1,10-3,53)$

$664(71,8 \%)$

$112(12,1 \%)$

$98(10,6 \%)$

$717(77,5 \%)$

$700(75,7 \%)$

$15036(1073-84316)$

0

1261241

$$
\begin{gathered}
14890(976-84307) \\
0 \\
1222189
\end{gathered}
$$

$103(75-135)$

$75(52-103)$

$26(2,8 \%)$

Table 3: Predictive factors of $A \cup C>0$ s of $>10 \mathrm{~min} \%$

\begin{tabular}{|l|c|c|c|} 
& \multicolumn{3}{|c}{ for $R$ hemishere } \\
\hline & $\boldsymbol{\beta}$ & $\mathbf{p}$ & OR $(95 \% \mathrm{CI})$ \\
\hline TIVA Propofol & 1,622 & 0,000 & $5,061(2,999-8,542)$ \\
\hline Age & 0,029 & 0,000 & $1,019(1,017-1,041)$ \\
\hline MMSE $_{\text {Preop }}$ & $-0,127$ & 0,001 & $0,881(0,815-0,952)$ \\
\hline Constant & 2,710 & 0,025 & \\
\hline
\end{tabular}

The Hosmer-Lemeshow test was valid :

$x^{2}=6,156, d f=8 ; p=0,630$

\section{Conclusions}

Our Results illustrate that patients' intrinsic factors increase the risk of intraoperative BS. This risk increases depending on the choice of anesthetics and despite an adequate level of anaesthesia (DOA kept between $40-60$ ). In order to minimize the occurrence of BS, the DOA should be guided by raw EEG and spectral analysis and not by predefined values.

\section{References}

1. Anesth Analg 2016;122:234-42

2, Anesth Analg 2015;120:649-52 